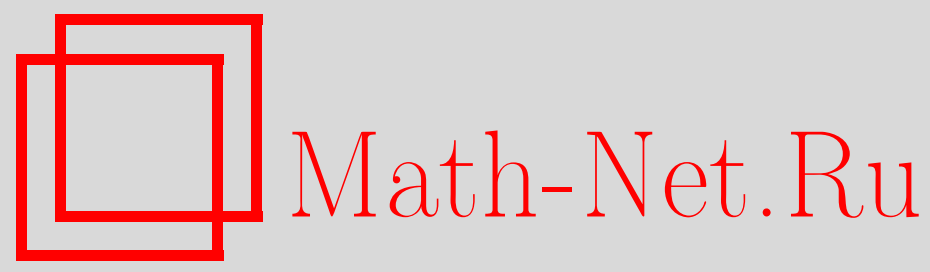

А. А. Финогенов, О конечных $p$-группах с циклическим коммутантом и циклическим центром, Матем. заметки, 1998, том 63, выпуск 6, 911-922

DOI: https://doi.org/10.4213/mzm1362

Использование Общероссийского математического портала Math-Net.Ru подразумевает, что вы прочитали и согласны с пользовательским соглашением http://www . mathnet.ru/rus/agreement

Параметры загрузки:

IP : 34.229 .108 .108

26 апреля 2023 г., $16: 23: 11$

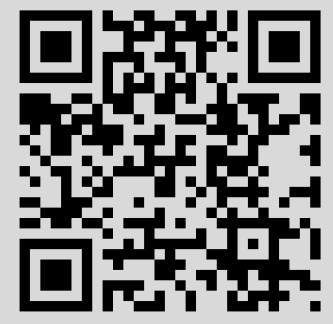




\section{О КОНЕЧНЫХ $p$-ГРУППАХ С ЦИКЛИЧЕСКИМ КОММУТАНТОМ И ЦИКЛИЧЕСКИМ ЦЕНТРОМ}

\section{А. А. Финогенов}

В работе описаны с точностью до изоморфизма конечные $p$-группы $G$ с циклическим коммутантом, циклическим центром и дополнительным ограничением: $\left[G^{\prime}, G\right] \leqslant G^{4}$ при $p=2$.

Библиограффия: 6 названий.

Работа посвящена описанию с точностью до изоморфизма конечных $p$-групп $G$ с циклическим коммутантом, циклическим центром и дополнительньм условием при $p=2$ : $\left[G^{\prime}, G\right] \leqslant G^{\prime 4}$. Эти группы представимы в виде центрального произведения 2-порожденной групшы одного из тринадцати типов, перечисленных в лемме 11 , с группй класса 2 (теорема 1). В теореме 2 приведены необходимые и достаточные условия изоморфизма таких групш.

Выбор именно этого класса групп объясняется тем, что, с одной стороны, описание болееширокого класса конечных $p$-групा - с циклическим коммутантом (и даже $p$-групा класса 2 с коммутантом порядка $p^{2}$ ) - сводится к сложной матричной задаче [1]. С другой стороны, более узкий класс групп - конечных $p$-групп класса 2 с циклическим центром - описан с точностью до изоморфизма Леонгом [2], [3] в 1974 и 1978 годах. Описание с точностью до изоморфизма 2 -групп $G$ с условием $\left[G^{\prime}, G\right] \nless G^{\prime 4}$ неизвестно, и вероятно оно окажется слишком громоздким [4].

В работе использованы стандартные обозначения [5].

Пусть $G-p$-группа с циклическим коммутантом, $a, b$ - элементы из $G$. При $p=2$ потребуем дополнительно $\left[G^{\prime}, G\right] \leqslant G^{\prime 4}$.

ЛЕмма $1[4$, лемма 1$] .|[b, a]|=\left|\left[b, a^{p}\right]\right| \cdot p=\left|\left[b^{p}, a\right]\right| \cdot p$.

ЛЕмма 2 [4, лемма 2]. Если $a, b, c$ - әлементы $G$ такие, что $|[a, b]| \geqslant|[b, c]|$, то $\left[b, a^{\alpha} c\right]=1$ для некоторого натурального $\alpha$.

ЛЕмма $3\left[4\right.$, предложение 2]. Если $\langle b, a\rangle^{\prime}=G^{\prime}$, mo $G=\langle a, b\rangle * C_{G}(\langle a, b\rangle)$.

ЛЕмма 4. Eсли $p=2$, mo $(a b)^{2^{k}}=a^{2^{k}} b^{2^{k}}[b, a]^{2^{k-1}} c$, əде $c \in\left\langle[b, a]^{2^{k}}\right\rangle$.

Лемма 5. Пусть $|a| \leqslant p^{s},|b| \leqslant p^{s}$ u, кроме того, $|[a, b]|<p^{s}$ nри $p=2$. Тогда $|a b| \leqslant p^{s}$.

Лемма 6. Пусть $p=2,|a|=p^{s},|b|=p^{s} u|[a, b]|=p^{s}$. Тогдa $|a b|=p^{s+1}$.

Лемма 7. Пусть $p=2,|a|=p^{s+1},|b|=p^{s} u|[a, b]|=p^{s}$. Тогдa $|a b|=p^{s}$. 
Лемма 8. Пусть $|a| \geqslant|b|, b^{p^{k}} \in\langle a\rangle$, но $b^{p^{k-1}} \notin\langle a\rangle$. Кроме того, пусть при $p=2|a|>p^{k+1}$. Тогда $\langle a\rangle \cap\left\langle a^{\alpha} b\right\rangle=1 u\left|a^{\alpha} b\right|=p^{k}$ для подходящего иелого $\alpha$.

ЛЕмма 9 [4, лемма 3]. Если $G$ - 2-порожденная p-группа класса не выше $2 u$ $\left|G^{\prime}: \gamma_{3}(G)\right|=p^{k}$, то в $p$-группе $G$ найдутся әлементы $a, b$ и с такие, что $G=$ $\langle a\rangle\langle b\rangle\langle c\rangle,[a, b]=b^{p^{k}} c,[a, c]=[b, c]=1$.

Лемма 10 [2]. Существует в точности четыре типа конечных 2-порохлденных p-групп класса не выше 2 с ииклическим иентром (а значит, и с ииклическим коммутантом):

1) $n p u 2 r \leqslant n u p \geqslant 2 Q(n, r)=\langle b\rangle \lambda\langle a\rangle, a^{p^{n}}=b^{p^{r}}=1, b^{p^{n-r}}=[a, b]$;

2) $n p u 2 r>n u p \geqslant 2 Q(n, r)=\langle a\rangle(\langle b\rangle \times\langle c\rangle), a^{p^{n}}=b^{p^{r}}=1, c=[a, b]$, $a^{p^{n-r}}=c^{p^{2 r-n}}$

3) $\operatorname{npu} p=2 \quad Q(n)=\langle a\rangle\langle c\rangle\langle b\rangle, a^{p^{n}}=b^{p^{n}}=c^{p^{n-1}},|c|=p^{n}, c=[a, b]$;

4) $Q(n, 0)$ - ииклическая $p$-группа порядка $p^{n}$.

ДокАЗАТЕЛЬСТво ЛЕмм. Лемму 4 докажем индукцией по $k$. При $k=1$, очевидно, $(a, b)^{2}=a^{2} b^{2}[b, a][b, a, b]$. Далее,

$$
\begin{aligned}
\left((a b)^{2^{k}}\right)^{2} & =\left(a^{2^{k}} b^{2^{k}}[b, a]^{2^{k-1}} c\right)^{2}=\left(a^{2^{k}} b^{2^{k}}[b, a]^{2^{k-1}}\right)^{2} c^{2} d \\
& =\left(a^{2^{k}} b^{2^{k}}\right)^{2}[b, a]^{2^{k}} e c^{2} d=a^{2^{k+1}} b^{2^{k+1}} f[b, a]^{2^{k}} e c^{2} d .
\end{aligned}
$$

Из леммы 1 следует, что $d, e$ и $f$ лежат в $\left\langle[b, a]^{2^{k+1}}\right\rangle$.

Если $p \neq 2$, то лемма 5 следует из регулярности $[5,3.10 .2]$ групп с циклическим коммутантом. При $p=2$ леммы 5 и 6 следуют непосредственно из леммы 4.

Для доказательства леммы 7 заметим, что из леммы 4 следует $(a b)^{2^{s}}=a^{2^{s}}[b, a]^{2^{s-1}}$. Так как $a^{2^{s}},[b, a]^{2^{s-1}} \in Z(G)$ и $\left|a^{2^{s}}\right|=\left|[b, a]^{2^{s-1}}\right|=2$, имеем $(a b)^{2^{s}}=1$.

При $p \neq 2$ лемма 8 доказана в [6, лемма 2.1.6]. В случае $p=2$ заметим, что в силу леммы $1|[a, b]| \leqslant 2^{k}$. Итак, возможны два случая: $|a|>|b|$ или $\left|a^{2^{k}}\right|=\left|b^{2^{k}}\right| \geqslant 4$. В первом случае найдется $\alpha$ такое, что $\left(a^{\alpha}\right)^{2^{k}}=\left(b^{-1}\right)^{2^{k}}$ и $|a|>\left|a^{\alpha}\right|=|b|=2^{k}$. По лемме $1\left|\left[a^{\alpha}, b\right]\right|<2^{k}$, поэтому ввиду леммы $4\left|a^{\alpha} b\right| \leqslant 2^{k}$. Так как $|[a, b]|=\left|\left[a, a^{\alpha} b\right]\right|$, из леммы 1 следует, что $\left|a^{\alpha} b\right|=2^{k}$ и $\langle a\rangle \cap\left\langle a^{\alpha} b\right\rangle=1$.

Во втором случае по лемме $4\left(a^{\alpha} b\right)^{2^{k+1}}=1$ для некоторого подходящего $\alpha$, т.е. $|a|>\left|a^{\alpha} b\right|$, и мы приходим к предыдущему случаю. Итак, лемма 8 доказана.

Лемма 11. Существует всего тринадиать типов конечных 2-порохсденых p-групп $G$ класса выше 2 с ииклическим иентром, ииклическим коммутантом порядка $p^{s}$ и двумя условиями:

1) $\left[G^{\prime}: \gamma_{3}(G)\right]=p^{k}$

2) $k>1$ при $p=2$.

A именно:

(1.a) $\langle b\rangle \lambda\langle a\rangle:|a|=p^{s},|b|=p^{s+k},[a, b]=b^{p^{k}}$;

(1.b) $\langle a\rangle\langle b\rangle:|a|=p^{s+m+k},|b|=p^{s+k}, a^{p^{s+m}}=b^{p^{s}},[a, b]=b^{p^{k}}, m<t$;

(1.c) $\langle a\rangle\langle b\rangle:|a|=p^{s+m+k},|b|=p^{s+k}, a^{p^{s+m}}=b^{p^{s}},[a, b]=b^{p^{k}}, m \geqslant t$;

(2.a) $(\langle b\rangle \times\langle c\rangle) \lambda\langle a\rangle:|a|=|b|=|c|=p^{s},[a, c]=[b, c]=1,[a, b]=b^{p^{k}} c$; 
(2.b) $(\langle b\rangle \times\langle c\rangle)\langle a\rangle:|a|=p^{s+s-m},|b|=|c|=p^{s}, a^{p^{s}}=c^{p^{m}},[a, c]=1,[b, c]=1$, $[a, b]=b^{p^{k}} c, s>m>k$ u npu $p=2 \quad s>m+1$;

(2.c) $(\langle b\rangle \times\langle c\rangle)\langle a\rangle:|a|=p^{s+s-m},|b|=|c|=p^{s}, a^{p^{s}}=c^{p^{m}},[a, c]=1,[b, c]=1$, $[a, b]=b^{p^{k}} c, k>m>0$

(3.a) $(\langle b\rangle\langle c\rangle) \lambda\langle a\rangle:|a|=p^{s},|b|=p^{s+l+k},|c|=p^{t+l+k},\left(b^{p^{k}} c\right)^{p^{t}}=c^{p^{t+l} \varphi},[a, c]=$ $[b, c]=1,[a, b]=b^{p^{k}} c$, əде $t=s-k, l>0 u \varphi \not \equiv 0(\bmod p)$

(3.b) $\langle a\rangle\langle b\rangle\langle c\rangle:|a|=p^{s+l+s-m},|b|=p^{s+l+k},|c|=p^{t+l+k}, a^{p^{s}}=c^{p^{m}},\left(b^{p^{k}} c\right)^{p^{t}}=c^{p^{t+l} \varphi}$, $[a, c]=[b, c]=1,[a, b]=b^{p^{k}} c$, əде $t=s-k, l>0, t>m>0 u \varphi \not \equiv 0(\bmod p)$

(4.a) $(\langle b\rangle\langle c\rangle) \lambda\langle a\rangle:|a|=p^{s},|b|=p^{s+k-l},|c|=p^{s},\left(b^{p^{k}} c\right)^{p^{t}}=c^{p^{t}} c^{p^{t+l} \varphi},[a, c]=[b, c]=1$, $[a, b]=b^{p^{k}} c$, əде $t=s-k, k>l>0 u \varphi \not \equiv 0(\bmod p)$;

(4.b) $\langle a\rangle\langle b\rangle\langle c\rangle:|a|=p^{s-l+s-m},|b|=p^{s+k-l},|c|=p^{s},\left(b^{p^{k}} c\right)^{p^{t}}=c^{p^{t}} c^{p^{t+l} \varphi}, a^{p^{s}}=c^{p^{m}}$, $[a, c]=[b, c]=1,[a, b]=b^{p^{k}} c$, где $t=s-k, k>l>0, t>m>0 u$ $\varphi \not \equiv 0(\bmod p)$

(5.a) $(\langle b\rangle\langle c\rangle) \lambda\langle a\rangle:|a|=p^{s},|b|=p^{s+k},|c|=p^{s-l},\left(b^{p^{k}} c\right)^{p^{t}}=c^{p^{t-l} \varphi},[a, c]=[b, c]=1$, $[a, b]=b^{p^{k}} c$, əдe $|z|=p^{k}, t=s-k, t>l \geqslant 0, \varphi \not \equiv 0(\bmod p) ;$

(5.b) $\langle a\rangle\langle b\rangle\langle c\rangle:|a|=p^{s+s-l-m},|b|=p^{s+k},|c|=p^{s-l},\left(b^{p^{k}} c\right)^{p^{t}}=c^{p^{t+l} \varphi}, a^{p^{s}}=c^{p^{m}}$, $[a, c]=[b, c]=1,[a, b]=b^{p^{k}} c$, əдe $|z|=p^{k}, t=s-k, t>l \geqslant 0, t-l>m>0$, $\varphi \not \equiv 0(\bmod p)$

(6.a) $(\langle b\rangle\langle c\rangle)\langle a\rangle:|a|=|b|=2^{s+1},|c|=2^{s},[a, c]=[b, c]=1,[a, b]=b^{2^{k}} c, a^{2^{s}}=b^{2^{s}}=$ $c^{2^{s-1}}=[a, b]^{2^{s-1}}$.

Kроме того, у групп типа (*.a) $\exp (G)=\exp \left(C_{G}\left(G^{\prime}\right)\right)=|b|$, за исключением групп тuпа (2.a), у которых $\exp (G)=p^{s+1}$, a $\exp \left(C_{G}\left(G^{\prime}\right)\right)=|b|=p^{s} . \quad$ У груnп muna (*.b) $\exp (G)=|a|$, y zpynn muna (*.a) $u(* . \mathrm{b}) \exp \left(C_{G}\left(G^{\prime}\right)\right) \cdot p^{t}>\exp (G)$ u y zpynn muna $(* . c) \quad \exp \left(C_{G}\left(G^{\prime}\right)\right) \cdot p^{t} \leqslant \exp (G)$.

ДокАЗАТЕльСтво. Строение метациклических $p$-групп, в том числе и с циклическим центром, хорошо известно (это группы типа (1)), поэтому будем считать, что $G$ не метациклическая.

В силу леммы $9 G=\langle a\rangle\langle b\rangle\langle c\rangle,[a, b]=b^{p^{k}} c,[a, c]=[b, c]=1$ и $\left|b^{p^{k}} c\right|=p^{s}$.

Докажем сначала, что $Z(G)=\langle c\rangle$. Действительно, если $a^{\alpha} b^{\beta} c^{\gamma} \in Z(G)$, то $a^{\alpha} b^{\beta} \in$ $Z(G)$. Тогда $1=\left[a, a^{\alpha} b^{\beta}\right]=\left[a, b^{\beta}\right]$, т.е. $b^{\beta} \in Z(G)$. Поскольку $Z(G)$ - циклическая группа, то либо $\langle c\rangle \leqslant\left\langle b^{\beta}\right\rangle$, либо $\left\langle b^{\beta}\right\rangle \leqslant\langle c\rangle$. В первом случае $G=\langle a\rangle\langle b\rangle$ и, очевидно, $G$ метациклическая. Значит, $\left\langle b^{\beta}\right\rangle \leqslant\langle c\rangle$. С другой стороны, $1=\left[b, b^{\alpha} a^{\beta}\right]$ и либо $\left\langle a^{\alpha}\right\rangle \geqslant\langle c\rangle$, либо $\left\langle a^{\alpha}\right\rangle \leqslant\langle c\rangle$.

Докажем, что в первом случае $G$ метациклическая. Пусть $\bar{b}=b a^{2^{t} \beta}$. Тогда $[a, \bar{b}]=$ $[a, b]=b^{2^{k}} c$. Но $\bar{b}^{2^{k}}=b^{2^{k}} a^{2^{s} \beta}$. Так как $a^{2^{s-1}} \notin Z(G)$ и $\langle a\rangle \geqslant\langle c\rangle$, существует $\beta$ такое, что $a^{2^{s}} \beta=c,[a, \bar{b}]=\bar{b}^{2^{k}}$ и $\langle a, \bar{b}\rangle$ - метациклическая група.

Итак, мы доказали, что $a^{\alpha} b^{\beta} c^{\gamma} \in\langle c\rangle$ и $\langle c\rangle=Z(G)$.

1) Рассмотрим случай $\langle b\rangle \cap\langle c\rangle=1$. Так как по лемме $1 b^{p^{s}} \in Z(G)=\langle c\rangle$, то $|b|=p^{s}$ и $p^{s}=\left|G^{\prime}\right|=\left|b^{p^{k}} c\right|=|c|$.

Из леммы 1 следует, что $a^{p^{s}} \in Z(G)=\langle c\rangle$. Ясно, что существуют целые числа $\alpha$ и $m$ такие, что $\bar{c}=c^{\alpha},\langle c\rangle=\langle\bar{c}\rangle$ и $a^{p^{s}}=\bar{c}^{p^{m}}$.

Так как $\left[a, b^{\alpha}\right]=\left(b^{p^{k}} c\right)^{\alpha}=\left(b^{\alpha}\right)^{p^{k}} c^{\alpha}$, группа $H=\langle a\rangle\langle\bar{b}\rangle\langle\bar{c}\rangle$, где $\bar{b}=b^{\alpha}$, является 
группой типа (2.a) (если $m=0)$, типа (2.b) (если $0>m>k)$, типа (2.c) (если $k \geqslant m)$ и $H=G$. Так как $G$ не метациклическая, имеет место $m \neq s$.

Если $p=2$ и $s-m=1$, то, применив лемму 7 , получим $m=0$ и, следовательно, $G$ - группа типа (2.a). Лемма 6 показьвает, что экспонента групп типа (2.a) при $p=2$ равна $2^{s+1}$.

2) Рассмотрим случай $\langle b\rangle \cap\langle c\rangle \neq 1$ и $|b|=|c| \cdot p^{k}$. Как уже отмечалось, $b^{p^{s}} \in Z(G)=\langle c\rangle$ и, значит, $\left(b^{p^{k}} c\right)^{p^{t}} \in\langle c\rangle$. А так как $\left|G^{\prime}\right|=p^{s}$, имеем $\left|\left(b^{p^{k}} c\right)^{p^{t}}\right|=p^{k}$ и $\left(b^{p^{k}} c\right)^{p^{t}}=c^{p^{t+l} \varphi}$ для некоторого $\varphi \not \equiv 0(\bmod p),|b|=p^{s+l+k}$ и $|c|=p^{t+l+k}$, где $l$ - некоторое неотрицательное число. Кроме того, $a^{p^{s}} \in Z(G)=\langle c\rangle$. Если $a^{p^{s}} \in\left\langle c^{p^{t}}\right\rangle=\left\langle b^{p^{s}}\right\rangle$, то по лемме 8 $\left\langle a b^{\alpha}\right\rangle \cap\langle b, c\rangle=1$ для некоторого $\alpha$. Следовательно, $\langle b, c\rangle \lambda\left\langle a b^{\alpha}\right\rangle$ является группой типа (3.а) в случае $l>0$ и группой типа (5.а) в случае $l=0$. Если $a^{p^{s}} \notin\left\langle c^{p^{t}}\right\rangle=\left\langle b^{p^{s}}\right\rangle$, то существуют $\alpha$ и $m$ такие, что $\bar{c}=c^{\alpha},\langle c\rangle=\langle\bar{c}\rangle$ и $a^{p^{s}}=\bar{c}^{p^{t-m}}$. Аналогично 1$)$, легко проверить, что $G=\langle a\rangle\langle\bar{b}\rangle\langle\bar{c}\rangle$, где $\bar{b}=b^{\alpha}$, является группй типа (3.b) в случае $l>0$ и группой типа (5.a) в случае $l=0$.

3) Рассмотрим случай $\langle b\rangle \cap\langle c\rangle \neq 1,|b|<|c| \cdot p^{k}$. Заметим, что $\left(b^{p^{k}} c\right)^{p^{t}}=c^{p t} b^{p^{s}}$ и $b^{p^{s}}$ лежит в собственной подгруппе $\left\langle c^{p t}\right\rangle$. Следовательно, $|c|=\left|b^{p^{k}} c\right|=p^{s}$. Отсюда вытекает, что $|b|=p^{s+k-l}$ для некоторого натурального $l$, и существует $\varphi \not \equiv 0(\bmod p)$ такое, что $c^{p^{t+l} \varphi}=b^{p^{s}}$.

Как уже отмечалось в 1 ), заменяя $c$ и $b$ на $c^{\alpha}$ и $b^{\alpha}$, мы можем считать, что $a^{p^{s}}=c^{p^{r}}$ для некоторого натурального $r$.

Если $l<r<l+t$, то $G$ - группа типа (4.b) и $m=r-l$.

Если $r \geqslant l+t,|a|=2^{s+1}$ и $|b|=2^{s+1}$ то, очевидно, $a^{2^{s}}=b^{2^{s}}=c^{2^{s-1}}=[a, b]^{2^{s-1}}$ и $G$ - группа типа (6.a).

Если $r \geqslant l+t$, но $|a| \neq 2^{s+1}$ или $|b| \neq 2^{s+1}$, то $c^{p^{r}} \in\left\langle b^{p^{s}}\right\rangle$. В силу леммы $5\left\langle a b^{\beta}\right\rangle \cap\langle c\rangle$ для некоторого натурального $\beta$, и $\langle b, c\rangle \lambda\left\langle a b^{\beta}\right\rangle$ - групша типа (4.a).

Если $r \leqslant l$, то по лемме 8 для некоторого натурального $\alpha\left\langle b\left(a^{p^{t}}\right)^{\alpha}\right\rangle \cap\langle b\rangle=1$, $\left[a, b\left(a^{p^{t}}\right)^{\alpha}\right]=b^{p^{k}} c=\left(b a^{\alpha}\right)^{p^{k}} a^{-\alpha p^{t}} c$ и $a^{-\alpha p^{t}} \in\langle c\rangle$. Значит, $\langle a\rangle\left(\left\langle b\left(a^{p^{t}}\right)^{\alpha}\right\rangle \times\left\langle a^{-\alpha p^{t}} c\right\rangle\right)-$ группа типа (2.c).

4) Осталось рассмотреть случай $\langle b\rangle \cap\langle c\rangle \neq 1$ и $|c| \cdot p^{k}<|b|$. Очевидно, $\left(b^{p^{k}} c\right)^{p^{t}}=b^{p^{s}} c^{p^{t}}$. Так как $\left\langle c^{p^{t}}\right\rangle<\left\langle b^{p^{s}}\right\rangle$, имеем $\left|G^{\prime}\right|=\left|b^{p^{k}} c\right|=\left|b^{p^{k}}\right|$. Отсюда $|b|=p^{s+k}$ и $|c|=p^{s-l}$ для некоторого подходящего неотрищательного $l$.

Рассуждения, относящиеся к элементу $a$, проводятся аналогично 2). Лемма доказана.

Легко заметить, что центральное произведение $A * B$ груп $A$ и $B$ с циклическими центрами $\widehat{A}$ и $\widehat{B}$ соответственно имеет циклический центр тогда и только тогда, когда центры "склеиваются", т.е. $\widehat{A}=\widehat{B}$. В дальнейшем мы будем рассматривать именно такие центральные произведения.

ПРЕДЛОЖЕНИЕ (см. [2], [3]). Конечная р-группа класса 2 с ииклическим иентром однозначно представима либо в виде иентрального произведения групп

$$
Q\left(n_{1}, r_{1}\right) * \cdots * Q\left(n_{\alpha}, r_{\alpha}\right) * Q(l, l)^{\varepsilon_{l}} * \cdots * Q(1,1)^{\varepsilon_{1}},
$$

әде $Q(n, r)$ - әруппа из леммы $10, Q(l, l)^{\varepsilon_{l}}$ есть иентральное произведение $\varepsilon_{l}$ әкземпляров $Q(l, l), \alpha \geqslant 0, \varepsilon_{i} \geqslant 0, i=1, \ldots, l, n_{1}>\cdots>n_{\alpha}>l, n_{\alpha}>r_{1}>\cdots>r_{\alpha} \geqslant 0$, 
$0<n_{1}-r_{1}<\cdots<n_{\alpha}-r_{\alpha}$, а при $p=2$ и $\alpha>0 ;$ либо в виде чентрального произведения групп

$$
Q(n) * Q(l, l)^{\varepsilon_{l}} * \cdots * Q(1,1)^{\varepsilon_{1}},
$$

где $n>l \geqslant 1, \varepsilon_{i} \geqslant 0, i=1, \ldots, l, u p=2$.

Теорема 1. Существует тринадиать типов конечных р-групп $P$ класса выше 2 с ииклическим коммутантом, ииклическим иентром и условиями

$$
\left|P^{\prime}\right|=p^{s}, \quad\left[P^{\prime}: \gamma_{3}(P)\right]=p^{k} .
$$

A именно, $P=G * D, Z(G) \subseteq D$ (что равносильно $D=C_{P}\left(G^{\prime}\right)$ ). Здесь конечная p-группа $D$ класса 2 с ииклическим иентром, $\left|D^{\prime}\right| \leqslant p^{k}$, представима в виде иентрального произведения групп $Q\left(n_{1}, r_{1}\right), \ldots, Q\left(n_{\alpha}, r_{\alpha}\right), Q\left(l_{1}, l_{1}\right), \ldots, Q\left(l_{\beta}, l_{\beta}\right)$, где $\alpha, \beta \geqslant 0, n_{1}>\cdots>n_{\alpha}, p^{k} \geqslant l_{1} \geqslant \cdots \geqslant l_{\beta}, p^{k} \geqslant r_{1}>\cdots>r_{\alpha}>0$, $n_{0}<n_{1}-r_{1}<\cdots<n_{\alpha}-r_{\alpha}$, zде $n_{0}$ maкое, что $\exp (G)=p^{n_{0}+s}$, а при $p=2$ $u \alpha>0 ; a G-$ одна из следующих групп $\left(v, u-\right.$ числа такие, что $p^{v}=\exp (D) u$ $\left.|c| \cdot p^{k-u}=\exp (D)\right)$ :

(A.a) zpynna muna (1.a) с условиями (1), $v \leqslant s$;

(A.b) zруппа типа (1.b) с условиями (1), $v \leqslant s+k$;

(A.c) zpynna muna (1.c) с условиями (1), $v \leqslant m+2 k$;

(B.a) zpynna muna (2.a) с условиями (1), $v \leqslant s$;

(B.b) zpynna muna (2.b) с условиями (1), $v \leqslant s$;

(B.c) zpynna muna (2.c) с условиями (1), $v \leqslant s+k-m$;

(C.a) zpynna muna (3.a) с условиями (1), $и>0$;

(C.b) zpynna muna (3.b) с условиями (1), $u>0$;

(D.a) zpynna muna (4.a) с условиям $и$ (1), $u \geqslant 0$;

(D.b) zpynna muna (4.b) с условиями (1), $u \geqslant 0$;

(E.a) zpynna muna (5.a) с условиями (1), $и>0$;

(E.b) zpynna muna (5.b) с условиями (1), $и>0$;

(F.a) zpynna muna (6.a) с условиям

Вообще говоря, центральное произведение, в отличие от прямого, определяется не только своими множителями, но и способом “склейки". Но в нашем случае все групш, кроме одной, имеют класс нильпотентности не вьше 2, поэтому, как сказано в [2], произведение таких групп однозначно (с точностью до изоморфизма) определяется своими сомножителями. Действительно, пусть, например, $P=G * D$ - группа с циклическим центром и циклическим коммутантом, $D=\langle a\rangle(\langle b\rangle \times\langle c\rangle)$ - групша класса $2,\langle c\rangle=Z(D)$ и $\langle z\rangle=Z(G)$. Можем считать, что $\bar{c}=z^{p^{r}}$ или $\bar{c}^{p^{r}}=z$, где $\bar{c}=c^{\alpha}, \alpha \neq \equiv 0(\bmod p)$. Но группа $\langle\bar{a}\rangle\langle b\rangle\langle\bar{c}\rangle$, где $\bar{a}=c^{\alpha}$, изоморфна $D$. Более того, сушествует изоморфизм, продолжающий отображение $a \mapsto \bar{a}, b \mapsto b$ и $c \mapsto \bar{c}$. (Случай $D=\langle a\rangle\langle b\rangle$ рассматривается аналогично.)

Таким образом, мы можем считать, что в центральном произведении групшы “подклеиваются" друг к другу некоторым единственным каноническим способом.

Теорема 2. Если $P$ и $\bar{P}$ - группь, удовлетворяющие условиям теоремы 1, $\bar{e}, \bar{m}, \bar{\varphi}-$ параметры группы $\bar{P}$, аналогичные соответствующим параметрам группьи $P$, то $P \cong \bar{P}$ тогда и только тогда, когда выполнены следуюшие условия:

1) $\bar{P}$ и $P$ - группы одного типа (в смысле теоремы 1); 
2) $\bar{m}=m$, если $\bar{P}$ u $P$ - zpynnы muna (*.b);

3) $\bar{l}=l$, если $\bar{P}$ u $P$ - әрyпnы muna (C), (D) uли (E);

4) $\bar{D} \cong D$;

5) $\bar{\varphi} \equiv \varphi\left(\bmod p^{\min (t, u)}\right)$, ecлu $\bar{P} u P-$ zpynnbl muna (C.a);

6) $\bar{\varphi} \equiv \varphi\left(\bmod p^{\min (m, u)}\right)$, если $\bar{P} u P-$ гpynnы muna (C.b);

7) $\bar{\varphi} \equiv \varphi\left(\bmod p^{\min (t, u)}\right)$, ecлu $\bar{P} u P-$ гpynnы muna (D.a);

8) $\bar{\varphi} \equiv \varphi\left(\bmod p^{\min (m, u)}\right)$, если $\bar{P} u P-$ zpynnы muna (D.b);

9) $\bar{\varphi} \equiv \varphi\left(\bmod p^{\min (t-l, u)}\right)$, ecлu $\bar{P} u P-$ zpynnbl muna (E.a);

10) $\bar{\varphi} \equiv \varphi\left(\bmod p^{\min (m, u)}\right)$, ecлu $\bar{P} u P-$ zpynnbl muna (E.b).

ДокаЗАТЕЛЬСТво теоремы 1 . По лемме $3 P=G * D$, где $D=C_{P}(G)$. Поскольку $D^{\prime} \subseteq G^{\prime} \cap C_{G}(a)=\left\langle\left(b^{p^{k}} c\right)^{p^{t}}\right\rangle$, то $\left|D^{\prime}\right| \leqslant p^{k}$. Если у групшы $P \exp \left(C_{P}\left(P^{\prime}\right)\right) \cdot p^{t} \leqslant \exp (P)$, то аналогичное условие выполнено и для $G$. Из леммы 11 следует, что $G$ - группа типа (1.c) или (2.c), значит $P$ - групша типа (А.c) или (В.c).

В случае, когда $\exp \left(C_{P}\left(P^{\prime}\right)\right) \cdot p^{t}>\exp (P)$, можем считать, что $|b| \geqslant \exp \left(C_{P}\left(G^{\prime}\right)\right)$. Действительно, если существует элемент $d \in C_{P}\left(G^{\prime}\right)$ и $|d|>|b|$, то по лемме 2 для некоторого натурального $\alpha\left[d b^{\alpha}, a\right]=1, b d b^{\alpha} \in C_{P}\left(G^{\prime}\right),\left[b d b^{\alpha}, a\right]=[b, a]$. Тогда, как легко проверить, группа $\langle a\rangle\left\langle b d b^{\alpha}\right\rangle\left\langle c\left(d b^{\alpha}\right)^{-p^{k}}\right\rangle$ удовлетворяет соотношениям леммы 9. Применяя преобразования из доказательства леммы 11 , можем привести эту группу к виду (1)-(6), при этом $|b|\left(\right.$ равньй $\left.\exp \left(C_{G}\left(G^{\prime}\right)\right)\right)$ не изменится.

Докажем, что если $G$ - группа типа $(\mathrm{C})$ или $(\mathrm{E})$, то $\exp (D)<|c| \cdot p^{k}$. Пусть $d \in D$ и $|d| \geqslant|c| \cdot p^{k}$. Рассмотрим групшу $\langle a\rangle\langle\bar{b}\rangle\langle\bar{c}\rangle$, где $\bar{b}=b d^{\alpha}$ и $\bar{c}=c\left(d^{-p^{k}}\right)^{\alpha}$. Поскольку

$$
[a, \bar{b}]=[a, b]=b^{p^{k}} c=\left(b d^{\alpha}\right)^{p^{k}} c\left(d^{\alpha}\right)^{-p^{k}}=\bar{b}^{p^{k}} \bar{c}
$$

$\langle a\rangle\langle\bar{b}\rangle\langle\bar{c}\rangle$ удовлетворяет соотношениям из леммы 9. Так как $\left|D^{\prime}\right| \leqslant p^{k}$, по лемме 1 $d^{-p^{k}} \in Z(P)$, значит $c \in\left\langle d^{-p^{k}}\right\rangle$. Следовательно, при подходящем $\alpha \bar{c}=1$ и $\langle a\rangle\langle\bar{b}\rangle\langle\bar{c}\rangle-$ метациклическая $p$-группа.

Докажем, что в группах типа (F.a) $\exp (D)<2^{s+1}$. Пусть существует $d \in D$ и $|d|=2^{s+1}$. Тогда $|a d|=2^{s}$ и $\langle a d, b, c\rangle$ - группа типа (4.a) и, значит, $G$ - группа типа (D.a).

Строение группы $D$ определяется леммой 9 и следующими шестью леммами.

ЛЕМма 12. Если $n_{1} \geqslant n_{2} u n_{1}-r_{1} \geqslant n_{2}-r_{2}$, mo

$$
Q\left(n_{1}, r_{1}\right) * Q\left(n_{2}, r_{2}\right) \cong Q\left(n_{1}, r_{1}\right) * Q\left(r_{2}, r_{2}\right) .
$$

Лемма 13. Eсли $n<n_{1}$, mo $Q(n) * Q\left(n_{1}, r_{1}\right) \cong Q(n-1, n-1) * Q\left(n_{1}, r_{1}\right)$.

ЛЕмма 14. Eсли $p=2, \operatorname{mo} Q(n, n) * Q(n, n) \cong Q(n+1) * Q(n+1)$.

ЛЕмма 15. Если $p=2, \operatorname{mo} Q(n, n) \cong Q(n+1, n)$.

Леммы 12-15 доказаны в [2] и [3].

Лемма 16. Пусть $P=G * D$ - конечная $p$-группа с ииклическим иентром $и$ ииклическим коммутантом, $G^{\prime}=P^{\prime}, G$ - группа класса выше $2, D$ - группа muna $Q(n, r)$ (m.e. $D=\langle d, e\rangle$, əдe $|d|=n u|e|=r), p^{n+t} \leqslant \exp (G) u p^{n-r+s} \leqslant \exp (G)$ $(t, s$ и т из леммы 11). Тогда $P \cong \bar{G} * \bar{D}$, где $\bar{D}$ - әруппа типа $Q(r, r), \bar{G} \cong G u$ $\bar{G} \subseteq G\langle e\rangle$. 
ДоказАтельство. Сначала заметим, что $r<k=s-t$, так как $\left|D^{\prime}\right|=p^{r}, D^{\prime} \subseteq$ $Z(P) \cup G^{\prime}$ и $p^{n+t} \leqslant \exp (G)$.

Пусть $|a|=\exp (G)$. (Если $|b|=\exp (G)$, то необходимо поменять $a$ и $b$ местами.) Тогда $\left|a^{p^{s}}\right| \geqslant\left|d^{p^{r}}\right|$ и $\left|a^{p^{t}}\right| \geqslant|d|$. Положим $\bar{d}=d a^{p^{t} \alpha}$. Так как $d^{p^{r}} \in\left\langle a^{p^{s}}\right\rangle$ и $a^{p^{t}} \in C_{P}\left(P^{\prime}\right)$, по лемме 2 существует $\alpha$ такое, что $|\bar{d}|=p^{r}$; следовательно, $\langle\bar{d}, e\rangle=\bar{D}$ - групша типа $Q(r, r)$. Пусть $\bar{b}=b e^{\beta}$. Существует $\beta$ такое, что $[\bar{b}, \bar{d}]=1$. Действительно, так как $\left|a^{p^{s}}\right| \geqslant d$, имеем $\alpha \equiv 0\left(\bmod p^{k-t}\right)$, значит $|[\bar{d}, b]| \leqslant p^{r}$. Существование подходящего $\beta$ теперь следует из леммы 2 . Итак, если $\bar{D}=\langle\bar{d}, e\rangle$ и $\bar{G}=\langle a, \bar{b}\rangle$, то $G * D=\bar{G} * \bar{D}$. Поскольку $\left|e^{\beta}\right| \leqslant p^{k}$, отображение $a \mapsto a, b \mapsto \bar{b}$ продолжается до изоморфизма $G$ на $\bar{G}$. Лемма доказана.

Лемма 17. Если группа $G$ удовлетворяет условиям леммы $16, D$ - группа тиna $Q(n)$, mo $P=G * D \cong G * Q(n, n)$.

ДоказАТЕльСтво. Как и вьше, заменим $e$ на $\bar{e}=e a^{p^{t} \alpha}(b$ на $\bar{b})$, где $\alpha$ такое, что $|\bar{e}|<|e|$. Применив к группе $\langle d, \bar{e}\rangle$ лемму 7 , получим, что $\langle d, \bar{e}\rangle-$ группа типа $Q(n, n)$. Лемма доказана.

По лемме $9 D$ представима в виде центрального произведения групп $Q\left(n_{i}, r_{i}\right)$ и $Q(n)$. Используя леммы $12-17$, эти групшы можно преобразовать и переставить в требуемом порядке подобно тому, как это сделано в [2] и [3]. Теорема 1 доказана.

ДокаЗАТЕЛЬСТво теоРемы 2. 1) Сначала заметим, что групш типа (А.c) и (B.c) неизоморфны группам другого типа, так как лишш у этих групп $\exp (P)=\exp \left(C_{P}\left(P^{\prime}\right)\right) \cdot p^{t}$. Группы типа (А.c) неизоморфны группам типа (В.c), так как у (А.c) $\exp (P) \geqslant p^{s+k}$, y (B.c) $\exp (P)<p^{s+k}$.

Рассмотрим оставшиеся группы. Экспонента $\exp \left(C_{P}\left(P^{\prime}\right)\right)=\left\langle a^{p^{t}}\right\rangle\langle b\rangle\langle c\rangle D$ одинакова только у групп типа (A) и (Е) (в этом случае $\left.\exp \left(C_{P}\left(P^{\prime}\right)\right)=p^{s+k}\right)$ и у групп типа (D) и $(\mathrm{F})\left(\right.$ в этом случае $\exp \left(C_{P}\left(P^{\prime}\right)\right)=2^{s+1}$ и $p=2$ ). Но экспонента групшы типа (D.b) больше экспоненты групшы типа (F.a). Поэтому если группы разных типов и изоморфны, то это либо групшы типа (A) и (E), либо группы типа (D.a) и (F.a).

Докажем, что если группа $P$ типа $(\mathrm{A})$ изоморфна группе типа $(\mathrm{E})$, то у этой групы $|b|>\exp (D)$.

Допустим, это не так. Тогда существует элемент $d \in D$ такой, что $|d|=|b|$. Следовательно, $b^{p^{s}} \in\left\langle d^{p^{s}}\right\rangle$ и $\left|b d^{\alpha}\right|=p^{s}$ для некоторого натурального $\alpha$. Значит, в $P$ сушествуют элементы $x=b d^{\alpha}$ и $y=a$ такие, что

1) $x \in C_{P}\left(P^{\prime}\right), y \notin C_{P}\left(P^{\prime}\right)$ и $\langle[x, y]\rangle=P^{\prime}$;

2) $|x|=p^{s}$.

Однако, группа типа (Е) элементов с такими свойствами не содержит. Действительно, из условия 1) следует, что $x=b^{\alpha_{b}}\left(a^{p^{t}}\right)^{\alpha_{a}} c^{\alpha_{c}} d_{x}, y=a^{\beta_{a}} b^{\beta_{b}} c^{\beta_{c}} d_{y}$, где $d_{x}, d_{y} \in D$, и $\left\langle b^{\alpha_{b}}\right\rangle=\langle b\rangle,\left\langle a^{\beta_{a}}\right\rangle=\langle a\rangle$. Так как в групе типа (Е) порядок элемента $b$ больше, чем $\left|a^{p^{t}}\right|,|c|$ и $\exp (D)$, условие 2$)$ не может быть вьполнено.

Итак, пусть $P=\langle a\rangle\langle b\rangle * D$ - групша типа $(\mathrm{A})$, у которой $|b|>\exp (D)$, и в ней есть элементы $\bar{a}, \bar{b}, \bar{c}$ и подгрупша $\bar{D}$, для которых вьполняются условия типа (Е).

Заметим, что порядки элементов $a$ и $\bar{a}$ одинаковы, так как если $\exp (P)=\exp \left(C_{P}\left(P^{\prime}\right)\right)$, то $|a|=|\bar{a}|=p^{s}$, и если $\exp (P)>\exp \left(C_{P}\left(P^{\prime}\right)\right)$, то $|a|=|\bar{a}|=\exp (P)$. Порядки элементов $b$ и $\bar{b}$ также одинаковы и равны $\exp \left(C_{P}\left(P^{\prime}\right)\right)$, так как $|b|>\exp (D)$. Для получения противоречия достаточно показать, что $\exp (\bar{D}) \geqslant|\bar{c}| \cdot p^{k}$. 
Так как $a, \bar{a} \in P \backslash C_{P}\left(P^{\prime}\right), b, \bar{b} \in C_{P}\left(P^{\prime}\right)$ и $\langle[\bar{a}, \bar{b}]\rangle=P^{\prime}$, то $\bar{a}=a^{\alpha} y, y \in\langle b, D\rangle$, $\bar{b}=b^{\beta} x$, где $x \in\left\langle a^{p^{t}}, D\right\rangle$ и $\alpha, \beta \not \equiv 0(\bmod p)$.

С одной стороны, $[\bar{a}, \bar{b}]=\bar{b}^{p^{k}} \bar{c}$; с другой стороны, $[\bar{a}, \bar{b}] \equiv\left[a^{\alpha}, b^{\beta}\right]=[a, b]^{\beta f(\alpha)}=$ $\left(b^{p^{k}}\right)^{\beta f(\alpha)}=\left(\left(b^{\beta} x\right)^{p^{k}} x^{-p^{k}}\right)^{f(\alpha)}=\left(\bar{b}^{p^{k}} x^{-p^{k}}\right)^{f(\alpha)}\left(\bmod \left\langle\left[b, a^{p^{t}}\right]\right\rangle\right),\left[b, a^{p^{t}}\right]=b^{p^{s}} \in$ $P^{\prime} \cap Z(P)$ и $f(\alpha) \not \equiv 0(\bmod p)$. Поскольку $x^{p^{k}} \in Z(P)$, отсюда следует, что $\left(\bar{b}^{p^{k}}\right)^{1-f(\alpha)} \in$ $Z(P) \cap\langle\bar{b}\rangle=\left\langle b^{p^{s}}\right\rangle$ и $\bar{c} \in\left\langle x^{p^{k}}\right\rangle\left\langle b^{p^{s}}\right\rangle=\left\langle a^{p^{s}}\right\rangle D^{p^{k}}\left\langle b^{p^{s}}\right\rangle$, значит $|\bar{c}| \cdot p^{k} \leqslant \exp (D)$. Что и требовалось доказать.

Докажем, что групп типа (D.a) и (F.a) неизоморфны. Для этого заметим, что элемент $a$ в группе типа (D.a) обладает следуюшими свойствами: $|a|=p^{s},\left|\left[a, C\left(P^{\prime}\right)\right]\right|=p^{s}$; в группе типа (F.a) элементов с такими свойствами нет. Действительно, пусть $\bar{a}=$ $a^{\alpha} b^{\beta} d(d \in\langle c, D\rangle)$ - элемент групшы типа (F.a), обладающий этими свойствами. Поскольку $\left|\left[\bar{a}, C\left(P^{\prime}\right)\right]\right|=p^{s}$, то $\alpha$ нечетное. По лемме $4\left(\bar{a}^{\alpha} b^{\beta} d\right)^{2^{s}}=\bar{a}^{2^{s} \alpha} b^{2^{s} \beta}[b, a]^{2^{s-1} \alpha \beta}=$ $\bar{a}^{2^{s} \alpha}\left(b^{2^{s}}[b, a]^{2^{s-1}}\right)^{\beta}=\bar{a}^{2^{s} \alpha} \neq 1$. Получили противоречие.

2) Как отмечалось выше, порядки элементов $a$ и $\bar{a}$ одинаковы. Таким образом, в группах типа (А) и (В) $\bar{m}=m$. Для групп типа $(\mathrm{C})-(\mathrm{E})$ докажем, что $\bar{l}=l$; следовательно, $\bar{m}=m$.

3) В группах типа $(\mathrm{C}) \exp \left(C_{P}\left(P^{\prime}\right)\right)=|b|=p^{k+l+s}$, в группах типа (D) $\exp \left(C_{P}\left(P^{\prime}\right)\right)=$ $|b|=p^{s+k-l}$. Следовательно, в этих группах $\bar{l}=l$.

Пусть $P=\langle a\rangle\langle b\rangle\langle c\rangle * D$ - группа типа (C), (D) или (Е). Допустим, в $P$ можно выбрать элементы $\bar{a}, \bar{b}, \bar{c}$ и подгруппу $\bar{D}$ так, что для них вьполняются условия одного из типов (C), (D) или (Е). Так как $a, \bar{a} \in P \backslash C_{P}\left(P^{\prime}\right), b, \bar{b} \in C_{P}\left(P^{\prime}\right)$ и $\langle[\bar{a}, \bar{b}]\rangle=P^{\prime}$, можем считать, что $\bar{a}=a^{\alpha} b^{r} d_{1}$ и $\bar{b}=b^{\beta} x$, где $\alpha, \beta \not \equiv 0(\bmod p), x=a^{p^{t} \psi} d_{2}$ и $d_{1}, d_{2} \in D$. В этом случае

$$
\begin{aligned}
\bar{b}^{p^{k}} \bar{c} & =[\bar{a}, \bar{b}]=[a, b]^{\beta f(\alpha)}\left[b, a^{p^{t}}\right]^{r \psi}\left[d_{1}, d_{2}\right]=\left(b^{p^{k}} c\right)^{\beta f(\alpha)}\left[b, a^{p^{t}}\right]^{r \psi}\left[d_{1}, d_{2}\right] \\
& =\left(\left(b^{\beta} x\right)^{p^{k}}\left(x^{-p^{k}} c^{\beta}\right)\right)^{f(\alpha)}\left[b, a^{p^{t}}\right]^{r \psi}\left[d_{1}, d_{2}\right]=\bar{b}^{p^{k} f(\alpha)}\left(x^{-p^{k}} c^{\beta}\right)^{f(\alpha)}\left[b, a^{p^{t}}\right]^{r \psi}\left[d_{1}, d_{2}\right],
\end{aligned}
$$

где $f(\alpha)$ - число такое, что $\left[a^{\alpha}, b\right]=[a, b]^{\alpha}($ в частности, $f(\alpha) \not \equiv 0(\bmod p))$. Так как $\left(x^{-p^{k}} c^{\beta}\right)^{f(\alpha)}\left[b, a^{p^{t}}\right]^{r \psi}\left[d_{1}, d_{2}\right] \in Z(P)$, то $\bar{b}^{p^{k}(f(\alpha)-1)} \in Z(P) \cap\langle\bar{b}\rangle=\left\langle b^{p^{s}}\right\rangle$ (т.е. $\left.f(\alpha)-1 \equiv 0\left(\bmod p^{t}\right)\right)$ и

$$
\begin{gathered}
\bar{c}=\left(x^{-p^{k}} c^{\beta}\right)^{f(\alpha)}\left[b, a^{p^{t}}\right]^{r \psi} \bar{b}^{p^{k}(f(\alpha)-1)}\left[d_{1}, d_{2}\right], \\
\bar{b}^{p^{k}} \bar{c}=\left(b^{p^{k}} c\right)^{\beta f(\alpha)}\left[b, a^{p^{t}}\right]^{r \psi}\left[d_{1}, d_{2}\right] .
\end{gathered}
$$

Вернемся к доказательству теоремы. Вычислив порядок элементов в формуле (2), легко понять, что в группах типа (Е) $|\bar{c}|=|c|$, и (поскольку $\left.|c|=p^{k+t-l}\right) \bar{l}=l$. Что и требовалось доказать.

4) Докажем более сильное утверждение.

Лемма 18. Пусть $P=G * D \cong \bar{P}=\bar{G} * \bar{D}$ - әруппьи, удовлетворяющие условиям теоремь $2,\left\{n_{i}, \ldots, r_{i}, \ldots, l_{i}, \ldots\right\}$ - параметры группь $D u\left\{\bar{n}_{i}, \ldots, \bar{r}_{i}, \ldots, \bar{l}_{i}, \ldots\right\}-$ параметры группы $\bar{D}$. Тогда $n_{i}=\bar{n}_{i}, r_{i}=\bar{r}_{i}, l_{i}=\bar{l}_{i}$. Иными словами, множество пар чисел $\left\{\left(n_{i}, r_{i}\right), \ldots,\left(l_{j}, l_{j}\right), \ldots\right\}$ является инвариантом группы $P$. 
ДоКАЗАТЕЛЬСТВО. Построим процедуру, позволяющую определить пары чисел $\left\{\left(n_{i}, r_{i}\right), \ldots,\left(l_{j}, l_{j}\right), \ldots\right\}$ из абстрактного строения группы $P$.

Из лемм 1-10 следует, что $P / Z(P) \cong(\langle\widehat{b}\rangle \lambda\langle\widehat{a}\rangle) \times A$, где $A$ - абелева $p$-группа с инвариантами $\left(r_{1}, r_{1}, r_{2}, r_{2}, \ldots, l_{1}, l_{1}, l_{2}, l_{2}, \ldots\right)$ и $[\widehat{a}, \widehat{b}]=\widehat{b}^{p^{k}}$. Профакторизовав группу $P / Z(P)$ по коммутанту, получим абелеву $p$-группу с инвариантами $\left(s, k, r_{1}, r_{1}, r_{2}, r_{2}\right.$, $\left.\ldots, l_{1}, l_{1}, l_{2}, l_{2}, \ldots\right)$ и $s>k>r_{1}, \ldots, l_{1}, \ldots$.

Таким образом, множество $\left\{r_{1}, r_{2}, \ldots, l_{1}, l_{2}, \ldots\right\}$ определяется абстрактными свойствами групшы $P$.

Рассмотрим целочисленную функцию $f(g)$, равную максимальному среди чисел $f$ со свойством: существуют $x, y \in P$ такие, что $|x|=p^{f},|[x, y]|=p^{g}$ и для любого $y^{\prime} \in P$ $\left|\left[x, y^{\prime}\right]\right| \leqslant p^{g}$. Докажем, что $f\left(r_{i}\right)=n_{i}$ и $f\left(l_{j}\right) \leqslant h_{j}$, где $h_{j}$ - наибольшее число из множества $\left\{n_{i}-\left(r_{i}-l_{j}\right) \mid r_{i} \geqslant l_{j}, i \geqslant 1\right\} \cup\left\{n_{i}-\left(r_{i}-l_{j}\right) \mid r_{i} \geqslant l_{j}, i \geqslant 1\right\} \cup\{w\}$, a $w$ определяется из равенства $p^{w+s-l_{j}}=\exp (P)$.

Обозначим образующие группы $Q\left(n_{i}, r_{i}\right)$ через $a_{i}$ и $b_{i}$, а образующиегруппы $Q\left(l_{j}, l_{j}\right)$ через $c_{j}$ и $d_{j}$ (предполагается, что $\left|a_{i}\right|=p^{n_{i}},\left|b_{i}\right|=p^{r_{i}},\left|c_{i}\right|=\left|d_{i}\right|=p^{l_{i}}$ и $a_{i}, b_{i}, c_{j}, d_{j}$ удовлетворяют соответствующим соотношениям из леммы 10).

Заметим, что $f\left(r_{i}\right) \geqslant n_{i}$. Для этого нужно взять $a_{i}$ и $b_{i}$ в качестве $x$ и $y$.

Вообще говоря,

$$
x \equiv a^{\alpha} b^{\beta} \cdots a_{i}^{\alpha_{i}} \cdots\left(\bmod \left\langle b_{i}, \ldots, c_{i}, \ldots, d_{i}, \ldots\right\rangle\right)
$$

и если $|x|>p^{n_{i}}$, то порядок одного из трех элементов: $a_{k}^{\alpha_{k}}(k<i), a^{\alpha}$ или $b^{\beta}-$ больше $p^{n_{i}}$. Взяв в качестве $y^{\prime}$ соответственно $b_{k}, b$ или $a$, получим $\left|\left[x, y^{\prime}\right]\right|>p^{r_{i}}$. Следовательно, $f\left(r_{i}\right)=n_{i}$. Если $f\left(l_{j}\right)>h_{j}$, из формулы (4) следует, что либо $\left|a_{i}^{\alpha_{i}}\right|>p^{n_{i}-\left(r_{i}-l_{j}\right)}$ $\left(\right.$ при $r_{i} \geqslant l_{j}$ ) и в этом случае $\left|\left[a_{i}^{\alpha_{i}}, b_{i}\right]\right|>p^{l_{i}}$; либо $\left|a^{\alpha}\right|>p^{w}$ или $\left|b^{\beta}\right|>p^{w}$ и в этом случае $\left|\left[a^{\alpha}, b\right]\right|>p^{l_{i}}$ или $\left|\left[b^{\beta}, a\right]\right|>p^{l_{i}}$. Что противоречит определению $f(g)$.

Итак, множество пар $T=\left\{\left(n_{1}, r_{1}\right), \ldots,\left(f\left(l_{1}\right), l_{1}\right), \ldots\right\}$ является инвариантом группы $P$. Осталось найти способ отделять пары $\left(n_{1}, r_{1}\right), \ldots$ от пар $\left(f\left(l_{1}\right), l_{1}\right), \ldots$

Назовем пару $(x, y) \in T$ неустойчивой, если выполняется одно из условий:

1) существует пара $(\bar{x}, \bar{y}) \in T$ такая, что $(x, y) \neq(\bar{x}, \bar{y}), \bar{x} \geqslant x$ и $\bar{x}-\bar{y} \geqslant x-y$;

2) $p^{x+t} \leqslant \exp (G)$ и $p^{x-y+s} \leqslant \exp (G)(t$ и $s-$ параметры групшы $G)$.

Из определения чисел $h_{i}$ следует, что пара $(x, y) \in T$ устойчива (т.е. не является неустойчивой) тогда и только тогда, когда $(x, y)=\left(n_{i}, r_{i}\right)$ для некоторого $i$. Выбрасьвая из множества устойчивых пар повторяющиеся, мы получим в точности $\left\{\left(n_{1}, r_{1}\right), \ldots\right\}$. Лемма доказана.

5), 6) Шаг 1. Пусть теперь $P=\langle a\rangle\langle b\rangle\langle c\rangle * D$ и $\bar{P}=\langle\bar{a}\rangle\langle\bar{b}\rangle\langle\bar{c}\rangle * \bar{D}$ - изоморфные группы типа $(\mathrm{C}), \bar{m}=m$ и $\bar{l}=l$. Отождествив $P$ и $\bar{P}$, можно выбрать элементы $\bar{a}$, $\bar{b}, \bar{c}$ и подгруппу $\bar{D}$ так, что $\bar{a}=a^{\alpha} b^{r} d_{1}$ и $\bar{b}=b^{\beta} x$, где $x=a^{p^{t} \psi} d_{2}$ и $d_{1}, d_{2} \in D$. Из формул $(2),(3)$ и соотношений $\left(b^{p^{k}} c\right)^{p^{t}}=c^{p^{t+l} \varphi}$ и $\left(\bar{b}^{p^{k}} \bar{c}\right)^{p^{t}}=\bar{c}^{p^{t+l} \bar{\varphi}}$ следует, что

$$
\left(\bar{b}^{p^{k}} \bar{c}\right)^{p^{t}}=\left(b^{p^{k}} c\right)^{\beta f(\alpha) p^{t}}\left[b, a^{p^{t}}\right]^{r \psi p^{t}}\left[d_{1}, d_{2}\right]^{p^{t}}=c^{p^{t+l} \varphi \beta f(\alpha)}\left[b, a^{p^{t}}\right]^{r \psi p^{t}}\left[d_{1}, d_{2}\right]^{p^{t}}
$$

И

$$
\bar{c}^{p^{t+l} \bar{\varphi}}=x^{-p^{k} f(\alpha) p^{t+l} \bar{\varphi}} c^{\beta f(\alpha) p^{t+l} \bar{\varphi}}\left[b, a^{p^{t}}\right]^{r \psi p^{t+l} \bar{\varphi}} \bar{b}^{p^{k}(f(\alpha)-1) p^{t+l} \bar{\varphi}}\left[d_{1}, d_{2}\right]^{p^{t+l} \bar{\varphi}} .
$$


Отсюда

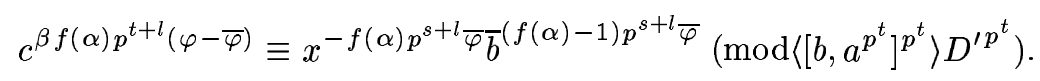

Шаг 2. Вычислив порядок элементов в (5) (учитывая, что $\left.f(\alpha)-1 \equiv 0\left(\bmod p^{t}\right)\right)$, легко понять, что если $\bar{\varphi} \not \equiv \varphi\left(\bmod p^{\min (m, t, u)}\right)$, то порядок левой части $(5)$ больше порядка правой, а это невозможно. Заметим, что если $P$ - группа типа (C.b), то $m<t$, a если (С.а), то $a^{p^{m}}=1$, т.е. $m$ исключается из рассуждений. Таким образом, мы доказали, что если $\bar{P}$ и $P$ изоморфны, то $\bar{\varphi} \equiv \varphi\left(\bmod p^{\min (t, u)}\right)$ или $\bar{\varphi} \equiv \varphi\left(\bmod p^{\min (m, u)}\right)$.

Шаг 3. Теперь докажем, что если $\bar{\varphi} \equiv \varphi\left(\bmod p^{\min (t, u)}\right)$ и $\bar{P}, P-$ групшы типа (С.а) или $\bar{\varphi} \equiv \varphi\left(\bmod p^{\min (m, u)}\right)$ и $\bar{P}, P$ - группы типа $(\mathrm{C} . \mathrm{b})$, то $\bar{P}$ и $P$ изоморфны.

Сначала найдем в $P$ элементы $\widehat{a}, \widehat{b}$ и $\widehat{c}$ такие, что $\langle\widehat{a}, \widehat{b}, \widehat{c}\rangle$ изоморфна $\bar{G}$. Для этого необходимо найти числа $\alpha, \beta, r, \psi$ (возможно равные 1 ) такие, что если положить $\widehat{a}=a^{\alpha} b^{r} d_{1}$, $\widehat{b}=b^{\beta} a^{p^{t} \psi} d_{2}$, где $\alpha, \beta \neq 0(\bmod p), d_{1}, d_{2} \in D$, и определить $\widehat{c}$ из равенства $[\widehat{a}, \widehat{b}]=\widehat{b}^{p^{k}} \widehat{c}$, то будут вьполнятся условия

$$
\widehat{a}^{p^{s}}=\widehat{c}^{p^{m}}
$$

если $P$ - группа типа (С.b), или $|\widehat{a}|=p^{s}$, если $P$ - группа типа (С.a), и

$$
\left(\widehat{b}^{p^{k}} \widehat{c}\right)^{p^{t}}=\widehat{c}^{p^{t+l} \bar{\varphi}}
$$

Кроме того, если $\alpha \neq 1$, то для корректного определения элемента $\widehat{c}$ необходимо, чтобы $[\widehat{a}, \widehat{b}] \cdot \widehat{b}^{-p^{k}} \in\langle\widehat{c}\rangle$. Рассуждая, как и при вьводе формулы $(2)$, получаем, что для этого достаточно вьполнения

$$
f(\alpha)-1 \equiv 0\left(\bmod p^{t}\right)
$$

где $f(\alpha)$ - число такое, что $\left[a^{\alpha}, b\right]=[a, b]^{f(\alpha)}$.

Из леммы 18 следует, что $C_{P}(\langle\widehat{a}, \widehat{b}, \widehat{c}\rangle) \cong D \cong \bar{D}$. Таким образом, группа $\langle\widehat{a}\rangle\langle\widehat{b}\rangle\langle\widehat{c}\rangle *$ $C_{P}(\langle\widehat{a}, \widehat{b}, \widehat{c}\rangle)=P$ действительно изоморфна групе $\bar{P}$.

Пусть сначала $\min (m, t, u)=u$ и $\widehat{a}=a, \widehat{b}=b^{\beta} d^{r}$, где $d$ - элемент максимального порядка из $D$.

Рассуждая так же, как и в доказательстве формулы (5), получаем, что из равенства

$$
c^{\beta p^{t+l}(\varphi-\bar{\varphi})}=\left(d^{r}\right)^{-p^{s+l} \bar{\varphi}}
$$

следует (7). Если $P$ - групша типа (С.a), то подходящие $r$ и $\beta$ существуют, так как $\left|c^{p^{t+l}(\varphi-\bar{\varphi})}\right|=\left|\left(d^{r}\right)^{p^{s+l}}\right|$.

Если $P$ - группа типа (C.b), то кроме (7) необходимо доказать и (6). Заметим, что (6) следует из $c^{p^{m}}=\widehat{c}^{p^{m}}$, которое, в свою очередь, следует из равенства

$$
c^{p^{m}(\beta-1)}=d^{r p^{k+m}}
$$

(для типа (C) $\left|c^{p^{m}}\right|>\left|d^{p^{k+m}}\right|$ ), так как согласно (2) $\widehat{c}=d^{-r p^{k}} c^{\beta}$. Существование подходящих $r$ и $\beta$ (что равносильно существованию “решения системы уравнений" $(9),(10)$ ) обеспечивает следующая 
Лемма 19. Если $a, b, c, d, e$ - әлементы одной ииклической $p$-группь, $|a|>|b|$, $|d| \geqslant|c|, \gamma \not \equiv 0(\bmod p)$, то существуют числа $r$ и $\beta$ такие, что $\beta \not \equiv 0(\bmod p)$, $a^{\beta-\gamma}=b^{r}, c^{\beta}=d^{r}$.

ДокАЗАТЕЛЬСТво леммы проведем индукцией по $|b|$. Если $|b|=0$, то $\beta=\gamma$, а $r$ легко найти из второго уравнения. Пусть $|b| \neq 0$ и $|a|=p^{i}$. По предположению индукции "уравнение" $a^{\beta-\gamma} \equiv b^{r}(\bmod \langle z\rangle)$, где $z \in\langle a\rangle$ и $|z|=p$, "имеет решение". Следовательно, существует число $\alpha$ такое, что $a^{\bar{\beta}-\gamma}=b^{r}$, где $\bar{\beta}=\beta+\alpha p^{i-1}$, и число $\bar{r} \equiv r\left(\bmod p^{i-1}\right)$ такое, что $c^{\bar{\beta}} \cdot d^{\bar{r}}=e$. Так как $|b|<p^{i-1}$, выполняется $a^{\bar{\beta}-\gamma}=b^{\bar{r}}$. Что и требовалось доказать.

Продолжим доказательство теоремы 2.

Шаг 4. Если $\min (m, t, u)=m$ и $\bar{P}, P$ - группы типа (C.b), то повторяем предыдущие рассуждения, полагая $a^{p^{t}}$ вместо $d$.

Шаг 5. Пусть теперь $\min (t, u)=t$ (значит, $t<k$ ), $\bar{P}_{\text {и }} P$ - группы типа (С.а) и $\widehat{a}=a^{\alpha}, \widehat{b}=b$, где $\alpha \not \equiv 0(\bmod p)$. Как и вьше, необходимо найти $\alpha$ такое, что выполняются (7) и (8). Рассуждая так же, как и в доказательстве формулы (5), получаем, что из равенства

$$
c^{f(\alpha) p^{t+l}(\varphi-\bar{\varphi})}=\widehat{b}^{(f(\alpha)-1) p^{s+l} \bar{\varphi}}
$$

следует (7). Заметим, что $\left|c^{p^{t+l}(\varphi-\bar{\varphi})}\right| \leqslant\left|\widehat{b}^{p^{s+l}}\right|=p^{k}$ и $f(\alpha) \equiv \alpha\left(\bmod p^{k}\right)$, так как $\left[b, a^{\alpha}\right]=[b, a]^{\alpha}[b, a, a]^{\left(\begin{array}{c}\alpha \\ 2\end{array}\right)} \ldots[4]$. Поэтому соотношение (11) можно заменить на

$$
c^{\alpha p^{t+l}(\varphi-\bar{\varphi})}=\widehat{b}^{(\alpha-1) p^{s+l} \bar{\varphi}} .
$$

Для вьполнения $(12)$ подходящее $\alpha$, очевидно, существует. При этом $\alpha-1 \equiv 0\left(\bmod p^{t}\right)$, а так как $t<k$, имеет место $f(\alpha)-1 \equiv 0\left(\bmod p^{t}\right)$.

$7), 8)$ Групшы типа (D) рассматриваются аналогично. На первом шаге, предполагая, что $\bar{a}=a^{\alpha} b^{r} d_{1}$ и $\bar{b}=b^{\beta} x$, где $x=a^{p^{t} \psi} d_{2}$ и $d_{1}, d_{2} \in D$ в силу (2), (3) и $\left(b^{p^{k}} c\right)^{p^{t}}=c^{p^{t}} c^{p^{t+l} \varphi}$, получаем

$$
\begin{gathered}
\left(\bar{b}^{p^{k}} \bar{c}\right)^{p^{t}}=c^{p^{t}} c^{\left(p^{t+l} \varphi\right) \beta f(\alpha)}\left[b, a^{p^{t}}\right]^{r \psi p^{t}}\left[d_{1}, d_{2}\right]^{p^{t}}, \\
\bar{c}^{p^{t}}=x^{-p^{k} f(\alpha) p^{t}} c^{\beta f(\alpha) p^{t}}\left[b, a^{p^{t}}\right]^{r \psi p^{t}} \bar{b}^{p^{k}(f(\alpha)-1) p^{t}}\left[d_{1}, d_{2}\right]^{p^{t}}, \\
\bar{c}^{p^{t+l} \bar{\varphi}}=x^{-p^{k} f(\alpha) p^{t+l} \bar{\varphi}} c^{\beta f(\alpha) p^{t+l} \bar{\varphi}}\left[b, a^{p^{t}}\right]^{r \psi p^{t+l} \bar{\varphi} \bar{b}^{p^{k}}(f(\alpha)-1) p^{t+l} \bar{\varphi}}\left[d_{1}, d_{2}\right]^{p^{t+l} \bar{\varphi}} .
\end{gathered}
$$

Отсюда и из $\left(\bar{b}^{p^{k}} \bar{c}\right)^{p^{t}}=\bar{c}^{p^{t}} \bar{c}^{p^{t+l} \bar{\varphi}}$ следует

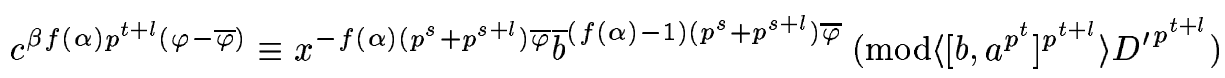

(это аналог формулы (5)).

На втором шаге, вычислив порядок элементов в $(13)\left(\right.$ у учетом $\left.f(\alpha)-1 \equiv 0\left(\bmod p^{t}\right)\right)$, получим, что из $\bar{\varphi} \not \equiv \varphi\left(\bmod p^{\min (m, t, u)}\right)$ следует $\bar{P} \not P$.

На третьем шаге, взяв $\widehat{a}=a, \widehat{b}=b^{\beta} d^{r}$, где $d$ - элемент максимального порядка из $D$, найдем $r$ и $\beta$ такие, что выполняется

$$
\widehat{a}^{p^{s}}=\widehat{c}^{p^{m+l}}
$$


если $P$ типа (D.b), и

$$
\left(\widehat{b}^{p^{k}} \widehat{c}\right)^{p^{t}}=\widehat{c}^{p^{t}} \widehat{c}^{p^{t+l} \bar{\varphi}}
$$

Рассуждая, как и при выводе формулы (13), заключаем, что (15) следует из

$$
c^{\beta p^{t+l}(\varphi-\bar{\varphi})}=d^{-\left(p^{s}+p^{s+l}\right) \bar{\varphi}},
$$

и если $P$ - группа типа (D.a), то подходящие $r$ и $\beta$, очевидно, существуют. Если же $P$ группа типа (D.b), то необходимо доказать и выполнение (14), что следует из

$$
c^{p^{m+l}(\beta-1)}=d^{r p^{k+m+l}} .
$$

Тогда существование подходящих $r$ и $\beta$ обеспечивает лемма 19.

На четвертом шаге повторяем предыдущие рассуждения, полагая $a^{p^{t}}$ вместо $d$.

На пятом шаге положим $\widehat{a}=a^{\alpha}, \widehat{b}=b$. Рассуждая, как и при выводе формулы (13), получаем, что (15) следует из

$$
c^{f(\alpha) p^{t+l}(\varphi-\bar{\varphi})}=\widehat{b}^{(f(\alpha)-1)\left(p^{s}+p^{s+l}\right) \bar{\varphi}} .
$$

Отсюда вытекает существование подходящего $\alpha$.

$9), 10)$ Групшы типа (Е) рассматриваются аналогично. Так как $\left(\bar{b}^{p^{k}} \bar{c}\right)^{p^{t}}=\bar{c}^{p^{t-l} \bar{\varphi}}$, вместо (5) нужно использовать

$$
c^{\beta f(\alpha) p^{t+l}(\varphi-\bar{\varphi})} \equiv x^{-f(\alpha) p^{s-l} \bar{\varphi} b}(f(\alpha)-1) p^{s-l} \bar{\varphi}\left(\bmod \left\langle\left[b, a^{p^{t}}\right]^{p^{t-l}}\right\rangle D^{\prime p^{t-l}}\right),
$$

вместо (7) - формулу $\left(\bar{b}^{p^{k}} \bar{c}\right)^{p^{t}}=\bar{c}^{p^{t-l} \bar{\varphi}}$, которая следует из $c^{\beta p^{t-l}(\varphi-\bar{\varphi})}=d^{-p^{s-l} \bar{\varphi}}$.

На пятом шаге роль $t$ играет $t-l$, а роль $(12)-c^{\alpha p^{t-l}(\varphi-\bar{\varphi})}=\bar{b}^{(\alpha-1) p^{s-l} \bar{\varphi}}$. Теорема доказана.

Автор выражает признательность В.В. Сергейчуку, привлекшему внимание к работе [1].

\section{СПИСОК ЦИТИРОВАННОЙ ЛИТЕРАТУРЫ}

[1] Сергейчук В.В. О классификации метабелевых $p$-групп // Матричные задачи. Киев: ИМ AH УССР, 1977. C. 150-161.

[2] Leong Y. K. Odd order nilpotent groups of class two with cyclic centre // J. Austral. Math. Soc. Ser. A. 1974. V. 17. P. 142-153.

[3] Leong Y. K. Finite 2-groups of class two with cyclic centre // J. Austral. Math. Soc. Ser. A. 1978. V. 27. P. $125-140$.

[4] Финогенов А. А. Конечные $p$-группы с циклическим коммутантом // Алгебра и логика. 1995. T. 34. № 2. C. 233-240.

[5] Huppert B. Endliche Gruppen. I. Grundlehren Math. Wiss. V. 134. Berlin-HeidelbergNew York: Springer, 1967.

[6] Cooper C. D. H. Power automorphisms of a group // Math. Z. 1968. V. 107. P. 335-336.

Институт математики и механики УрО РАН

E-mail : fin@top.imm.intec.ru 